\title{
On the weighted fractional Pólya-Szegö and Chebyshev-types integral inequalities concerning another function
}

\author{
Kottakkaran Sooppy Nisar ${ }^{1,2^{*}}$, Gauhar Rahman ${ }^{3}$, Dumitru Baleanu ${ }^{4,5,6}$, Muhammad Samraiz ${ }^{7}$ and \\ Sajid lqbal ${ }^{8}$
}

"Correspondence:

kottakkaran.sooppy.nisar@tdtu.edu.vn 'Informetrics Research Group, Ton Duc Thang University, Ho Chi Minh City, Vietnam

${ }^{2}$ Faculty of Mathematics and Statistics, Ton Duc Thang University, Ho Chi Minh City, Vietnam Full list of author information is available at the end of the article

\section{Springer}

\begin{abstract}
The primary objective of this present paper is to establish certain new weighted fractional Pólya-Szegö and Chebyshev type integral inequalities by employing the generalized weighted fractional integral involving another function $\Psi$ in the kernel. The inequalities presented in this paper cover some new inequalities involving all other type weighted fractional integrals by applying certain conditions on $\omega(\theta)$ and $\Psi(\theta)$. Also, the Pólya-Szegö and Chebyshev type integral inequalities for all other type fractional integrals, such as the Katugampola fractional integrals, generalized Riemann-Liouville fractional integral, conformable fractional integral, and Hadamard fractional integral, are the special cases of our main results with certain choices of $\omega(\theta)$ and $\Psi(\theta)$. Additionally, examples of constructing bounded functions are also presented in the paper.
\end{abstract}

MSC: 26A33; 26D10;26D15; 26D53;05A30

Keywords: Fractional integrals; Weighted fractional integrals; Inequalities; Chebyshev functional

\section{Introduction}

The field of integral inequalities plays an essential role in the diverse domain. The mathematicians have investigated that it is mainly a powerful tool for the improvement of both applied and pure mathematics. In [8], the authors established Grüss type integral inequalities by employing the classical fractional integrals. Certain new integral inequalities for the Riemann-Liouville (R-L) fractional integrals can be found in the work of Dahmani [6]. The inequalities involving an extension of the gamma function and confluent $k$-hypergeometric function were found in the work of Nisar et al. [26]. Nisar et al. [27] performed Gronwall inequalities with applications. Rahman et al. [42] gave certain inequalities for $(k, \rho)$-fractional integrals. Ostrowski type inequalities connecting local fractional integrals were found in [50]. Sarikaya et al. [51] developed generalized $(k, s)$-fractional integrals with applications. In [52], Set et al. introduced Grüss type inequalities by employing generalized $k$-fractional integrals. Recently, Nisar et al. [29] gave some new generalized fractional integral inequalities.

(c) The Author(s) 2020. This article is licensed under a Creative Commons Attribution 4.0 International License, which permits use sharing, adaptation, distribution and reproduction in any medium or format, as long as you give appropriate credit to the original author(s) and the source, provide a link to the Creative Commons licence, and indicate if changes were made. The images or other third party material in this article are included in the article's Creative Commons licence, unless indicated otherwise in a credit line to the material. If material is not included in the article's Creative Commons licence and your intended use is not permitted by statutory regulation or exceeds the permitted use, you will need to obtain permission directly from the copyright holder. To view a copy of this licence, visit http://creativecommons.org/licenses/by/4.0/. 
Very recently, the fractional conformable and proportional fractional integral operators have been given in [13, 15]. Later on, Huang et al. [12] gave Hermite-Hadamard type inequalities by using fractional conformable integrals (FCI). Qi et al. [33] investigated Čebyšev type inequalities involving FCI. The Chebyshev type inequalities and certain Minkowski type inequalities are found in [25, 30, 43]. Nisar et al. [28] investigated some new inequalities for a class of $n(n \in \mathbb{N})$ positive, continuous, and decreasing functions by employing FCI. Rahman et al. [41] introduced Grüss type inequalities for $k$-fractional conformable integrals. Some significant inequalities are given in [35-37, 39, 40, 46]. Very recently, Rahman et al. [38, 44] presented fractional integral inequalities involving tempered fractional integrals. In [2], Abdeljawad et al. presented some new local fractional inequalities associated with generalized $(s, m)$-convex functions and applications. Qi et al. [34] proposed fractional integral versions of Hermite-Hadamard type inequality for generalized exponential convexity. In [3], Abdeljawad et al. presented new fractional integral inequalities for p-convexity within interval-valued functions. Zhou et al. [55] investigated some new inequalities by considering the generalized proportional Hadamard fractional integral operators. Rashid et al. [48] proposed some inequalities via generalized proportional fractional integrals. In [47], the authors presented reverse Minkowski's inequalities via generalized proportional fractional integrals. In [21], Mohammed and Abdeljawad proposed some modifications of fractional integral inequalities for convex functions. Abdeljawad et al. [1] presented modified conformable fractional integral inequalities of Hermite-Hadamard type with applications. Mohammed and Brevik [23] investigated a new version of Hermite-Hadamard for Riemann-Liouville fractional integrals. Mohammed and Abdeljawad [22] studied integral inequalities for generalized fractional integral with nonsingular kernels. Mohammed and Srikaya [24] proposed generalized fractional integral inequalities for twice differentiable functions.

In [5], the well-known Chebyshev functional for two integrable functions $\hbar_{1}$ and $\hbar_{2}$ on $\left[x_{1}, x_{2}\right]$ is given by

$$
\begin{aligned}
\mathcal{T}\left(\hbar_{1}, \hbar_{2}\right) & =\frac{1}{x_{2}-x_{1}} \int_{x_{1}}^{x_{2}} \hbar_{1}(\theta) \hbar_{2}(\theta) d \theta \\
& -\frac{1}{x_{2}-x_{1}}\left(\int_{x_{1}}^{x_{2}} \hbar_{1}(\theta) d \theta\right) \frac{1}{x_{2}-x_{1}}\left(\int_{x_{1}}^{x_{2}} \hbar_{2}(\theta) d \theta\right) .
\end{aligned}
$$

The functional $\mathcal{T}\left(\hbar_{1}, \hbar_{2}\right) \geq 0$ for the two synchronous functions $\hbar_{1}$ and $\hbar_{2}$ on $\left[x_{1}, x_{2}\right]$, i.e.,

$$
\left(\hbar_{1}(\vartheta)-\hbar_{1}(\zeta)\right)\left(\hbar_{2}(\vartheta)-\hbar_{2}(\zeta)\right) \geq 0
$$

for any $\vartheta, \zeta \in\left[x_{1}, x_{2}\right]$.

In $[4,7,9,17]$, the researchers studied functional (1.1) and introduced a large number of interesting integral inequalities. Very recently, Rahman et al. and Tassaddiq et al. [45, 53] studied functional (1.1) and investigated some new inequalities involving fractionally conformable.

The well-known Grüss [11] inequality for two integrable functions $\hbar_{1}$ and $\hbar_{2}$ on $\left[x_{1}, x_{2}\right]$ is given by

$$
\left|\mathcal{T}\left(\hbar_{1}, \hbar_{2}\right)\right| \leq \frac{\left(P_{1}-p_{1}\right)\left(Q_{1}-q_{1}\right)}{4},
$$


such that $\hbar_{1}$ and $\hbar_{2}$ fulfill the inequalities $p_{1} \leq \hbar_{1}(\vartheta) \leq P_{1}$ and $q_{1} \leq \hbar_{2}(\zeta) \leq Q_{1}$ for all $\vartheta, \zeta \in\left[x_{1}, x_{2}\right]$ and for some constant $p_{1}, q_{1}, P_{1}, Q_{1} \in \mathbb{R}$.

Pólya and Szegö [32] gave the following inequality:

$$
\frac{\int_{x_{1}}^{x_{2}} \hbar_{1}^{2}(\theta) d \theta \int_{x_{1}}^{x_{2}} \hbar_{2}^{2}(\theta) d \theta}{\left(\int_{x_{1}}^{x_{2}} \hbar_{1}(\theta) \hbar_{2}(\theta) d \theta\right)^{2}} \leq \frac{1}{4}\left(\sqrt{\frac{P_{1} Q_{1}}{p_{1} q_{1}}}+\sqrt{\frac{p_{1} q_{1}}{P_{1} Q_{1}}}\right)^{2} .
$$

Dragomir and Diamond [10] gave the following inequality with the help of Pólya-Szegö inequality:

$$
\left|\mathcal{T}\left(\hbar_{1}, \hbar_{2}\right)\right| \leq \frac{\left(P_{1}-p_{1}\right)\left(Q_{1}-q_{1}\right)}{4\left(x_{2}-x_{1}\right)^{2} \sqrt{p_{1} P_{1} q_{1} Q_{1}}} \int_{x_{1}}^{x_{2}} \hbar_{1}(\theta) d \theta \int_{x_{1}}^{x_{2}} \hbar_{2}(\theta) d \theta,
$$

where the functions $\hbar_{1}$ and $\hbar_{2}$ are positive and integrable on $\left[x_{1}, x_{2}\right]$ such that $\hbar_{1}$ and $\hbar_{2}$ satisfy the inequalities $p_{1} \leq \hbar_{1}(\vartheta) \leq P_{1}$ and $q_{1} \leq \hbar_{2}(\zeta) \leq Q_{1}$ for all $\vartheta, \zeta \in\left[x_{1}, x_{2}\right]$ and for some constant $p_{1}, q_{1}, P_{1}, Q_{1} \in \mathbb{R}$.

In [31], Ntouyas et al. investigated some new Pólya-Szegö and Chebyshev type inequalities by considering the $\mathrm{R}-\mathrm{L}$ fractional integrals.

This paper is composed as follows:

In Sect. 2, we mention some basic definitions. Certain new Pólya-Szegö type inequalities for the weighted fractional integrals concerning another function are presented in Sect. 3. In Sect. 4, we present some new generalized Chebyshev type inequalities for the weighted fractional integrals concerning another function. In Sect. 5, certain new particular cases in terms of weighted fractional integrals are discussed. An example of constructing bounding functions is considered in Sect. 6. The concluding remarks are presented in Sect. 7.

\section{Auxiliary results}

In this section, we present some basic definitions and mathematical preliminaries.

Lemma 2.1 ([11]) Assume that the functions $\hbar_{1}, \hbar_{2}:\left[x_{1}, x_{1}\right] \rightarrow \mathbb{R}$ are positive with $p_{1} \leq$ $\hbar_{1}(\vartheta) \leq P_{1}$ and $q_{1} \leq \hbar_{2}(\vartheta) \leq Q_{1}$ for all $\vartheta \in\left[x_{1}, x_{1}\right]$, then the following inequality holds:

$$
\begin{aligned}
& \left|\frac{1}{x_{2}-x_{1}} \int_{x_{1}}^{x_{2}} \hbar_{1}(\vartheta) \hbar_{2}(\vartheta) d \vartheta-\frac{1}{x_{2}-x_{1}} \int_{x_{1}}^{x_{2}} \hbar_{1}(\vartheta) d \vartheta \frac{1}{x_{2}-x_{1}} \int_{x_{1}}^{x_{2}} \hbar_{2}(\vartheta) d \vartheta\right| \\
& \quad \leq \frac{1}{4}\left(P_{1}-p_{1}\right)\left(Q_{1}-q_{1}\right),
\end{aligned}
$$

where the constants $P_{1}, p_{1}, q_{1}, Q_{1} \in \mathbb{R}$ and $\frac{1}{4}$ is the sharp of inequality 2.1 .

Definition $2.1([18,54])$ The function $\hbar_{1}$ is said to be in the space $L_{p, r}[0, \infty[$ if

$$
L_{p, r}\left[0, \infty\left[=\left\{\hbar_{1}:\left\|\hbar_{1}\right\|_{L_{p, r}[0, \infty[}=\left(\int_{r}^{s}\left|\hbar_{1}(\vartheta)\right|^{p} \vartheta^{r} d \vartheta\right)^{\frac{1}{p}}<\infty, 1 \leq p<\infty, r \geq 0\right\} .\right.\right.
$$

Applying $r=0$ on (2.2) gives

$$
L_{p}\left[0, \infty\left[=\left\{\hbar_{1}:\left\|\hbar_{1}\right\|_{L_{p}[0, \infty[}=\left(\int_{r}^{s}\left|\hbar_{1}(\vartheta)\right|^{p} d \vartheta\right)^{\frac{1}{p}}<\infty, 1 \leq p<\infty\right\} .\right.\right.
$$


Definition 2.2 ([16]) Let the function $\hbar_{1} \in L_{1}[0, \infty[$ and suppose that $\Psi$ is monotone, increasing, and positive on $\left[0, \infty\left[\right.\right.$ and having continuous derivative $\Psi^{\prime}$ on $[0, \infty[$ with $\Psi(0)=0$. Then $\hbar_{1}$ (the Lebesgue real-valued measurable function) defined on $[0, \infty[$ is in the space $X_{\Psi}^{p}(0, \infty),(1 \leq p<\infty)$ if

$$
\left\|\hbar_{1}\right\|_{X_{\Psi}^{p}}=\left(\int_{r}^{s}\left|\hbar_{1}(\vartheta)\right|^{p} \Psi^{\prime}(\vartheta) d \vartheta\right)^{\frac{1}{p}}<\infty, \quad 1 \leq p<\infty
$$

When $p \rightarrow \infty$, then

$$
\left\|\hbar_{1}\right\|_{X_{\Psi}^{\infty}}=\mathbf{e s s} \sup _{0 \leq \vartheta<\infty}\left[\Psi^{\prime}(\vartheta) \hbar_{1}(\varrho)\right]
$$

Clearly, the space $X_{\Psi}^{p}(0, \infty)$ matches with the space $L_{p}[0, \infty[$ if $\Psi(\vartheta)=\vartheta$ for $1 \leq p<\infty$ and in a similar way with the space $L_{p, r}[1, \infty[$ if $\Psi(\vartheta)=\ln \vartheta$ for $1 \leq p<\infty$.

Definition 2.3 ([20, 49]) The classical left- and right-sided R-L fractional integrals of order $\kappa>0$ are respectively defined by

$$
\left({ }_{x_{1}} \mathcal{R}^{\kappa} \hbar_{1}\right)(\theta)=\frac{1}{\Gamma(\kappa)} \int_{x_{1}}^{\theta}(\theta-\vartheta)^{\kappa-1} \hbar_{1}(\vartheta) d \vartheta, \quad x_{1}<\theta,
$$

and

$$
\left({ }_{x_{1}} \mathcal{R}^{\kappa} \hbar_{1}\right)(\theta)=\frac{1}{\Gamma(\kappa)} \int_{x_{1}}^{\theta}(\vartheta-\theta)^{\kappa-1} \hbar_{1}(\vartheta) d \vartheta, \quad x_{2}>\theta,
$$

where the gamma function is defined by $\Gamma(\kappa)=\int_{0}^{\infty} \tau^{\kappa-1} e^{-\tau} d \tau, \tau \in \mathbb{C}$, and $\Re(\tau)>0$.

Definition 2.4 $([20,49])$ The one-sided R-L fractional integral of order $\kappa>0$ is defined by

$$
\left(\mathcal{R}_{0}^{\kappa, \tau} \hbar_{1}\right)(\theta)=\left(\mathcal{R}^{\kappa, \tau} \hbar_{1}\right)(\theta)=\frac{1}{\Gamma(\kappa)} \int_{0}^{\theta}(\theta-\vartheta)^{\kappa-1} \hbar_{1}(\vartheta) d \vartheta .
$$

Definition $2.5([20,49])$ Let the function $\hbar_{1}:\left[x_{1}, x_{2}\right] \rightarrow \mathbb{R}$ be an integrable function, and assume that the function $\Psi$ is increasing and positive monotone on $\left(x_{1}, x_{2}\right]$ and having continuous derivative on $\left(x_{1}, x_{2}\right)$. Then the left- and right-sided generalized RiemannLiouville fractional integrals of a function $\hbar_{1}$ concerning another function $\Psi$ are respectively defined by

$$
\left({ }_{x_{1}}^{\Psi} \mathcal{R}^{\kappa} \hbar_{1}\right)(\theta)=\frac{1}{\Gamma(\tau)} \int_{x_{1}}^{\theta}(\Psi(\theta)-\Psi(\vartheta))^{\kappa-1} \Psi^{\prime}(\vartheta) \hbar_{1}(\vartheta) d \vartheta, \quad x_{1}<\theta
$$

and

$$
\left({ }^{\Psi} \mathcal{R}_{x_{2}}^{\kappa} \hbar_{1}\right)(\theta)=\frac{1}{\Gamma(\tau)} \int_{\theta}^{x_{2}}(\Psi(\vartheta)-\Psi(\theta))^{\kappa-1} \Psi^{\prime}(\vartheta) \hbar(\vartheta) d \vartheta, \quad \theta<x_{2},
$$

where $\kappa \in \mathbb{C}$ with $\Re(\kappa)>0$. 
Definition 2.6 ([14]) Let the function $\hbar_{1}$ be integrable $X_{\Psi}^{p}(0, \infty)$, and suppose that the function $\Psi$ is increasing positive and monotone on $[0, \infty$ [ and having continuous derivative on $[0, \infty$ [ with $\Psi(0)=0$. Then the generalized weighted (left-sided) R-L fractional integral of the function $\hbar_{1}$ concerning another function $\Psi$ in the kernel is

$$
\left({ }_{x_{1}}^{\Psi} \mathcal{R}_{\omega}^{\kappa} \hbar_{1}\right)(\theta)=\frac{\omega^{-1}(\theta)}{\Gamma(\kappa)} \int_{x_{1}}^{\theta}(\Psi(\theta)-\Psi(\vartheta))^{\kappa-1} \omega(\vartheta) \Psi^{\prime}(\vartheta) \hbar_{1}(\vartheta) d \vartheta, \quad x_{1}<\theta
$$

where $\kappa, \in \mathbb{C}$ with $\Re(\kappa)>0$.

Remark 2.1 The following new weighted fractional integrals can be easily obtained:

i. Applying Definition 2.8 for $\Psi(\theta)=\theta$, we get the following weighted R-L fractional integral:

$$
\left({ }_{x_{1}} \mathcal{R}_{\omega}^{\kappa} \hbar_{1}\right)(\theta)=\frac{\omega^{-1}(\theta)}{\Gamma(\kappa)} \int_{x_{1}}^{\theta}(\theta-\vartheta)^{\kappa-1} \omega(\vartheta) \hbar_{1}(\vartheta) d \vartheta, \quad x_{1}<\theta
$$

ii. Applying Definition 2.8 for $\Psi(\theta)=\theta$, we get the following weighted Hadamard fractional integral operator:

$$
\left({ }_{x_{1}} \mathcal{R}_{\omega}^{\kappa} \hbar_{1}\right)(\theta)=\frac{\omega^{-1}(\theta)}{\Gamma(\kappa)} \int_{x_{1}}^{\theta}(\ln \theta-\ln \vartheta)^{\kappa-1} \omega(\vartheta) \hbar_{1}(\vartheta) \frac{d \vartheta}{\vartheta}, \quad x_{1}<\theta
$$

iii. Applying Definition 2.8 for $\Psi(\theta)=\frac{\theta^{\eta}}{\eta}, \eta>0$, we obtain the following weighted Katugampola fractional integral:

$$
\left({ }_{x_{1}} \mathcal{R}_{\omega}^{\kappa, \eta} \hbar_{1}\right)(\theta)=\frac{\omega^{-1}(\theta)}{\Gamma(\kappa)} \int_{x_{1}}^{\theta}\left(\frac{\theta^{\eta}-\vartheta^{\eta}}{\eta}\right)^{\kappa-1} \omega(\vartheta) \hbar_{1}(\vartheta) \frac{d \vartheta}{\vartheta^{1-\eta}}, \quad x_{1}<\theta .
$$

Similarly, we can obtain another type of weighted fractional integrals.

Remark 2.2 The following new weighted fractional integrals can be easily obtained:

i. Applying Definition 2.8 for $\omega(\theta)=1$ and $\Psi(\theta)=\theta$, we get (2.4);

ii. Applying Definition 2.8 for $\Psi(\theta)=\theta$, it will reduce to the left-sided generalized Riemann-Liouville fractional integral operator (2.6);

iii. Applying Definition 2.8 for $\omega(\theta)=\theta^{u}$ and $\Psi(\theta)=\ln \theta$, it will reduce to the left-sided Hadamard integral operator $[20,49]$;

iv. Applying Definition 2.8 for $\Psi(\theta)=\frac{\theta^{\eta}}{\eta}, \eta>0$, and $\omega(\theta)=1$, it will reduce to the left-sided Katugampola [18] fractional integral;

v. Applying Definition 2.8 for $\omega(\theta)=1$ and $\Psi(\theta)=\frac{\theta^{\alpha+s}}{\alpha+s}$ (where $\alpha \in(0,1], s \in \mathbb{R}$, and $\alpha+s \neq 0$ ), it reduces to the left-sided generalized FCI given by [19];

vi. Applying Definition 2.8 for $\omega(\theta)=1$ and $\Psi(\theta)=\frac{\left(\theta-x_{1}\right)^{\alpha}}{\alpha}, \alpha>0$, it reduces to the fractional conformable integral defined by Jarad et al. [15].

In this paper, we analyze the subsequent one-sided generalized weighted fractional integral.

Definition 2.7 Let the function $\hbar_{1}$ be integrable in the space $X_{\Psi}^{p}(0, \infty)$, and suppose that the function $\Psi$ is increasing, positive, and monotone on $[0, \infty[$ and having continuous 
derivative on $[0, \infty[$ with $\Psi(0)=0$. Then the one-sided generalized weighted fractional integral of the function $\hbar_{1}$ with respect to another function $\Psi$ in the kernel is given by

$$
\left({ }_{\omega}^{\Psi} \mathcal{R}_{0}^{\kappa} \hbar_{1}\right)(\theta)=\frac{\omega^{-1}(\theta)}{\Gamma(\kappa)} \int_{0}^{\theta}(\Psi(\theta)-\Psi(\vartheta))^{\kappa-1} \omega(\vartheta) \Psi^{\prime}(\vartheta) \hbar_{1}(\vartheta) d \vartheta
$$

Definition 2.8 For $0=\tau_{0}<\tau_{1}<\cdots<\tau_{p}<\tau_{p+1}=\tau$, we define the following sub-integrals for generalized weighted integral:

$$
\left({ }_{\omega}^{\Psi} \mathcal{R}_{\tau_{i}, \tau_{i+1}}^{\kappa} \hbar_{1}\right)(\tau)=\frac{\omega^{-1}(\theta)}{\Gamma(\kappa)} \int_{\tau_{i}}^{\tau_{i+1}}(\Psi(\tau)-\Psi(\vartheta))^{\kappa-1} \omega(\vartheta) \Psi^{\prime}(\vartheta) \hbar_{1}(\vartheta) d \vartheta
$$

Note that

$$
\begin{aligned}
\left({ }_{\omega}^{\Psi} \mathcal{R}_{0}^{\kappa} \hbar_{1}\right)(\tau)= & \sum_{i=0}^{p}{ }_{\omega}^{\Psi} \mathcal{R}_{\tau_{i}, \tau_{i+1}}^{\kappa}\left(\hbar_{1}\right)(\tau) \\
= & \frac{\omega^{-1}(\theta)}{\Gamma(\kappa)} \int_{0}^{\tau_{1}}(\Psi(\tau)-\Psi(\vartheta))^{\kappa-1} \omega(\vartheta) \Psi^{\prime}(\vartheta) \hbar_{1}(\vartheta) d \vartheta \\
& +\frac{\omega^{-1}(\theta)}{\Gamma(\kappa)} \int_{\tau_{1}}^{\tau_{2}}(\Psi(\tau)-\Psi(\vartheta))^{\kappa-1} \omega(\vartheta) \Psi^{\prime}(\vartheta) \hbar_{1}(\vartheta) d \vartheta+\cdots \\
& +\frac{\omega^{-1}(\theta)}{\Gamma(\kappa)} \int_{\tau_{p}}^{\tau}(\Psi(X)-\Psi(\vartheta))^{\kappa-1} \omega(\vartheta) \Psi^{\prime}(\vartheta) \hbar_{1}(\vartheta) d \vartheta
\end{aligned}
$$

Remark 2.3 If we set $\Psi(\tau)=\tau$ and $\omega(\theta)=1$, then (2.10) will reduce to the sub-integrals of $\mathrm{R}-\mathrm{L}$ fractional integral defined by [31].

\section{Some weighted fractional Pólya-Szegö type integral inequalities}

In this section, we present some new weighted fractional Pólya-Szegö type integral inequalities for positive and integrable functions by utilizing generalized weighted fractional integral (2.9) containing other function $\Psi$ in the kernel.

Lemma 3.1 Let the functions $\hbar_{1}$ and $\hbar_{2}$ be positive and integrable on $[0, \infty)$, and assume that the function $\Psi$ is increasing, positive, and monotone on $[0, \infty[$ and having continuous derivative on $\left[0, \infty\left[\right.\right.$ with $\Psi(0)=0$. Suppose that $f_{1}, f_{2}, g_{1}$, and $g_{2}$ are four positive and integrable functions on $[0, \infty)$ such that

$$
\begin{aligned}
\left(H_{1}\right) \quad 0<f_{1}(\vartheta) \leq \hbar_{1}(\vartheta) \leq f_{2}(\vartheta), \\
\\
0<g_{1}(\vartheta) \leq \hbar_{2}(\vartheta) \leq g_{2}(\vartheta), \quad \vartheta \in[0, \theta], \theta>0 .
\end{aligned}
$$

Then, for $\kappa>0$ and $\theta>0$, the following weighted fractional integral inequality holds:

$$
\frac{{ }_{\omega}^{\Psi} \mathcal{R}_{0}^{\kappa}\left(g_{1} g_{2} \hbar_{1}^{2}\right)(\theta)_{\omega}^{\Psi} \mathcal{R}_{0}^{\kappa}\left(f_{1} f_{2} \hbar_{2}^{2}\right)(\theta)}{\left({ }_{\omega}^{\Psi} \mathcal{R}_{0}^{\kappa}\left\{\left(f_{1} g_{1}+f_{2} g_{2}\right) \hbar_{1} \hbar_{2}\right\}(\theta)\right)^{2}} \leq \frac{1}{4} .
$$

Proof Utilizing the given hypothesis, we have

$$
\left(\frac{f_{2}(\vartheta)}{g_{1}(\vartheta)}-\frac{\hbar_{1}(\vartheta)}{\hbar_{2}(\vartheta)}\right) \geq 0
$$


Similarly, we have

$$
\left(\frac{\hbar_{1}(\vartheta)}{\hbar_{2}(\vartheta)}-\frac{f_{1}(\vartheta)}{g_{2}(\vartheta)}\right) \geq 0
$$

The product of (3.3) and (3.4) yields

$$
\left(\frac{f_{2}(\vartheta)}{g_{1}(\vartheta)}-\frac{\hbar_{1}(\vartheta)}{\hbar_{2}(\vartheta)}\right)\left(\frac{\hbar_{1}(\vartheta)}{\hbar_{2}(\vartheta)}-\frac{f_{1}(\vartheta)}{g_{2}(\vartheta)}\right) \geq 0
$$

From (3.5), it follows that

$$
\left(f_{1}(\vartheta) g_{1}(\vartheta)+f_{2}(\vartheta) g_{2}(\vartheta)\right) \hbar_{1} \hbar_{2}(\vartheta) \geq g_{1}(\vartheta) g_{2}(\vartheta) \hbar_{1}^{2}(\vartheta)+f_{1}(\vartheta) f_{2}(\vartheta) \hbar_{2}^{2}(\vartheta) .
$$

Now, multiplying (3.6) by $\frac{(\Psi(\theta)-\Psi(\vartheta))^{\kappa-1} \omega(\vartheta) \Psi^{\prime}(\vartheta)}{\Gamma(\kappa)}$ and integrating the resultant identity with respect to $\vartheta$ over $(0, \theta)$, we have

$$
\begin{aligned}
& \frac{1}{\Gamma(\kappa)} \int_{0}^{\theta}(\Psi(\theta)-\Psi(\vartheta))^{\kappa-1} \omega(\vartheta) \Psi^{\prime}(\vartheta)\left(f_{1}(\vartheta) g_{1}(\vartheta)+f_{2}(\vartheta) g_{2}(\vartheta)\right) \hbar_{1} \hbar_{2}(\vartheta) d \vartheta \\
& \geq \frac{1}{\Gamma(\kappa)} \int_{0}^{\theta}(\Psi(\theta)-\Psi(\vartheta))^{\kappa-1} \omega(\vartheta) \Psi^{\prime}(\vartheta) g_{1}(\vartheta) g_{2}(\vartheta) \hbar_{1}^{2}(\vartheta) d \vartheta \\
& \quad+\frac{1}{\Gamma(\kappa)} \int_{0}^{\vartheta}(\Psi(\theta)-\Psi(\vartheta))^{\kappa-1} \omega(\vartheta) \Psi^{\prime}(\vartheta) f_{1}(\vartheta) f_{2}(\vartheta) \hbar_{2}^{2}(\vartheta) d \vartheta
\end{aligned}
$$

Multiplying both sides of the above equation by $\omega^{-1}(\theta)$ and using Definition (2.9), we obtain

$$
{ }_{\omega}^{\Psi} \mathcal{R}_{0}^{\kappa}\left[\left(f_{1} g_{1}+f_{2} g_{2}\right) \hbar_{1} \hbar_{2}\right](\theta) \geq{ }_{\omega}^{\Psi} \mathcal{R}_{0}^{\kappa}\left(g_{1} g_{2} \hbar_{1}^{2}\right)(\theta)+{ }_{\omega}^{\Psi} \mathcal{R}_{0}^{\kappa}\left(f_{1} f_{2} \hbar_{2}^{2}\right)(\theta)
$$

Now, using the AM-GM inequality, i.e., $p_{1}+p_{2} \geq 2 \sqrt{p_{1} p_{2}}, p_{1}, p_{2} \mathbb{R}^{+}$, we get

$$
{ }_{\omega}^{\Psi} \mathcal{R}_{0}^{\kappa}\left[\left(f_{1} g_{1}+f_{2} g_{2}\right) \hbar_{1} \hbar_{2}\right](\theta) \geq 2 \sqrt{{ }_{\omega}^{\Psi} \mathcal{R}_{0}^{\kappa}\left(g_{1} g_{2} \hbar_{1}^{2}\right)(\theta)_{\omega}^{\Psi} \mathcal{R}_{0}^{\kappa}\left(f_{1} f_{2} \hbar_{2}^{2}\right)(\theta)} .
$$

It follows that

$$
{ }_{\omega}^{\Psi} \mathcal{R}_{0}^{\kappa}\left(g_{1} g_{2} \hbar_{1}^{2}\right)(\theta)_{\omega}^{\Psi} \mathcal{R}_{0}^{\kappa}\left(f_{1} f_{2} \hbar_{2}^{2}\right)(\theta) \leq \frac{1}{4}\left({ }_{\omega}^{\Psi} \mathcal{R}_{0}^{\kappa}\left[\left(f_{1} g_{1}+f_{2} g_{2}\right) \hbar_{1} \hbar_{2}\right](\theta)\right)^{2},
$$

which gives the required result (3.2).

Corollary 3.1 Let the functions $\hbar_{1}$ and $\hbar_{2}$ be positive and integrable on $[0, \infty)$, and assume that the function $\Psi$ is increasing, positive, and monotone on $[0, \infty[$ and having continuous derivative on $\left[0, \infty\left[\right.\right.$ with $\Psi(0)=0$. Suppose that $f_{1}, f_{2}, g_{1}$, and $f_{2}$ are four positive and integrable functions on $[0, \infty)$ such that

$$
\begin{aligned}
\left(H_{2}\right) \quad 0<p_{1} \leq \hbar_{1}(\vartheta) \leq P_{1}<\infty, \\
\\
0<q_{1} \leq \hbar_{2}(\vartheta) \leq Q_{1}<\infty, \quad \vartheta \in[0, \theta], \theta>0 .
\end{aligned}
$$


Then, for $\kappa>0$ and $\theta>0$, the following weighted fractional integral inequality holds:

$$
\frac{{ }_{\omega}^{\Psi} \mathcal{R}_{0}^{\kappa}\left(\hbar_{1}^{2}\right)(\theta)_{\omega}^{\Psi} \mathcal{R}_{0}^{\kappa}\left(\hbar_{2}^{2}\right)(\theta)}{\left({ }_{\omega}^{\Psi} \mathcal{R}_{0}^{\kappa}\left\{\hbar_{1} \hbar_{2}\right\}(\theta)\right)^{2}} \leq \frac{1}{4}\left(\sqrt{\frac{p_{1} q_{1}}{P_{1} Q_{1}}}+\sqrt{\frac{P_{1} Q_{1}}{p_{1} q_{1}}}\right)^{2} .
$$

Lemma 3.2 Let all the conditions of Lemma 3.1 be satisfied. Then, for $\kappa, \lambda>0$ and $\theta>0$, the following weighted fractional integral inequality holds:

$$
\frac{{ }_{\omega}^{\Psi} \mathcal{R}_{0}^{\kappa}\left(\hbar_{1}^{2}\right)(\theta)_{\omega}^{\Psi} \mathcal{R}_{0}^{\lambda}\left(\hbar_{2}^{2}\right)(\theta)_{\omega}^{\Psi} \mathcal{R}_{0}^{\kappa}\left(f_{1} f_{2}\right)(\theta)_{\omega}^{\Psi} \mathcal{R}_{0}^{\lambda}\left(g_{1} g_{2}\right)(\theta)}{\left.{ }_{\omega}^{\Psi} \mathcal{R}_{0}^{\kappa}\left(f_{1} \hbar_{1}\right)(\theta)_{\omega}^{\Psi} \mathcal{R}_{0}^{\lambda}\left(g_{1} \hbar_{2}\right)(\theta)+{ }_{\omega}^{\Psi} \mathcal{R}_{0}^{\kappa}\left(f_{2} \hbar_{1}\right)(\theta)_{\omega}^{\Psi} \mathcal{R}_{0}^{\lambda}\left(g_{2} \hbar_{2}\right)(\theta)\right)^{2}} \leq \frac{1}{4}
$$

Proof By using hypothesis $\left(H_{1}\right)$ given by (3.1), we have

$$
\left(\frac{f_{2}(\vartheta)}{g_{1}(\zeta)}-\frac{\hbar_{1}(\vartheta)}{\hbar_{2}(\zeta)}\right) \geq 0
$$

and

$$
\left(\frac{\hbar_{1}(\vartheta)}{\hbar_{2}(\zeta)}-\frac{f_{1}(\vartheta)}{g_{2}(\zeta)}\right) \geq 0
$$

It gives

$$
\left(\frac{f_{1}(\vartheta)}{g_{2}(\zeta)}+\frac{f_{2}(\vartheta)}{g_{1}(\zeta)}\right) \frac{\hbar_{1}(\vartheta)}{\hbar_{2}(\zeta)} \geq \frac{\hbar_{1}^{2}(\vartheta)}{\hbar_{2}^{2}(\zeta)}+\frac{f_{1}(\vartheta) f_{2}(\vartheta)}{f_{1}(\zeta) f_{2}(\zeta)}
$$

Multiplying (3.9) by $g_{1}(\zeta) g_{2}(\zeta) \hbar_{2}^{2}(\zeta)$, we have

$$
\begin{gathered}
f_{1}(\vartheta) \hbar_{1}(\vartheta) g_{1}(\zeta) \hbar_{2}(\zeta)+f_{2}(\vartheta) \hbar_{1}(\vartheta) g_{2}(\zeta) \hbar_{2}(\zeta) \\
\geq g_{1}(\zeta) g_{2}(\zeta) \hbar_{1}^{2}(\vartheta)+f_{1}(\vartheta) f_{2}(\vartheta) \hbar_{2}^{2}(\zeta) .
\end{gathered}
$$

Taking product of (3.10) with $\frac{(\Psi(\theta)-\Psi(\vartheta))^{\kappa-1} \omega(\vartheta) \Psi^{\prime}(\vartheta)}{\Gamma(\kappa)}$ and integrating the resultant inequality with respect to $\vartheta$ over $(0, \theta)$, we have

$$
\begin{aligned}
& g_{1}(\zeta) \hbar_{2}(\zeta) \frac{1}{\Gamma(\kappa)} \int_{0}^{\theta}(\Psi(\theta)-\Psi(\vartheta))^{\kappa-1} \omega(\vartheta) \Psi^{\prime}(\vartheta) f_{1}(\vartheta) \hbar_{1}(\vartheta) d \vartheta \\
& \quad+g_{2}(\zeta) \hbar_{2}(\zeta) \frac{1}{\Gamma(\kappa)} \int_{0}^{\theta}(\Psi(\theta)-\Psi(\vartheta))^{\kappa-1} \omega(\vartheta) \Psi^{\prime}(\vartheta) f_{2}(\vartheta) \hbar_{1}(\vartheta) d \vartheta \\
& \geq g_{1}(\zeta) g_{2}(\zeta) \frac{1}{\Gamma(\kappa)} \int_{0}^{\theta}(\Psi(\theta)-\Psi(\vartheta))^{\kappa-1} \omega(\vartheta) \Psi^{\prime}(\vartheta) \hbar_{1}^{2}(\vartheta) d \vartheta \\
& +\hbar_{2}^{2}(\zeta) \frac{1}{\Gamma(\kappa)} \int_{0}^{\theta}(\Psi(\theta)-\Psi(\vartheta))^{\kappa-1} \omega(\vartheta) \Psi^{\prime}(\vartheta) f_{1}(\vartheta) f_{2}(\vartheta) d \vartheta
\end{aligned}
$$

Multiplying the above inequality by $\omega^{-1}(\theta)$ and applying (2.9), we get

$$
\begin{gathered}
g_{1}(\zeta) \hbar_{2}(\zeta)_{\omega}^{\Psi} \mathcal{R}_{0}^{\kappa}\left(f_{1} \hbar_{1}\right)(\theta)+g_{2}(\zeta) \hbar_{2}(\zeta)_{\omega}^{\Psi} \mathcal{R}_{0}^{\kappa}\left(f_{2} \hbar_{1}\right)(\theta) \\
\geq g_{1}(\zeta) g_{2}(\zeta)_{\omega}^{\Psi} \mathcal{R}_{0}^{\kappa}\left(\hbar_{1}^{2}\right)(\theta)+\hbar_{2}^{2}(\zeta)_{\omega}^{\Psi} \mathcal{R}_{0}^{\kappa}\left(f_{1} f_{2}\right)(\theta) .
\end{gathered}
$$


Again, taking product (3.11) with $\frac{\omega(\theta)(\Psi(\theta)-\Psi(\zeta))^{\lambda-1} \omega(\zeta) \Psi^{\prime}(\zeta)}{\Gamma(\lambda)}$ and integrating the resultant inequality with respect to $\zeta$ over $(0, \theta)$ and then applying (2.9), we get

$$
\begin{gathered}
{ }_{\omega}^{\Psi} \mathcal{R}_{0}^{\lambda}\left(g_{1} \hbar_{2}\right)(\theta)_{\omega}^{\Psi} \mathcal{R}_{0}^{\kappa}\left(f_{1} \hbar_{1}\right)(\theta)+{ }_{\omega}^{\Psi} \mathcal{R}_{0}^{\lambda}\left(g_{2} \hbar_{2}\right)(\theta){ }_{\omega}^{\Psi} \mathcal{R}_{0}^{\kappa}\left(f_{2} \hbar_{1}\right)(\theta) \\
\geq{ }_{\omega}^{\Psi} \mathcal{R}_{0}^{\lambda}\left(g_{1} g_{2}\right)(\theta){ }_{\omega}^{\Psi} \mathcal{R}_{0}^{\kappa}\left(\hbar_{1}^{2}\right)(\theta)+{ }_{\omega}^{\Psi} \mathcal{R}_{0}^{\lambda}\left(\hbar_{2}^{2}\right)(\theta)_{\omega}^{\Psi} \mathcal{R}_{0}^{\kappa}\left(f_{1} f_{2}\right)(\theta) .
\end{gathered}
$$

By using the AM-GM inequality, we get

$$
\begin{aligned}
& { }_{\omega}^{\Psi} \mathcal{R}_{0}^{\lambda}\left(g_{1} \hbar_{2}\right)(\theta){ }_{\omega}^{\Psi} \mathcal{R}_{0}^{\kappa}\left(f_{1} \hbar_{1}\right)(\theta)+{ }_{\omega}^{\Psi} \mathcal{R}_{0}^{\lambda}\left(g_{2} \hbar_{2}\right)(\theta){ }_{\omega}^{\Psi} \mathcal{R}_{0}^{\kappa}\left(f_{2} \hbar_{1}\right)(\theta) \\
& \quad \geq 2 \sqrt{\left.\left.\left.{ }_{\omega}^{\Psi} \mathcal{R}_{0}^{\lambda}\left(g_{1} g_{2}\right)(\theta)\right)_{\omega}^{\Psi} \mathcal{R}_{0}^{\kappa}\left(\hbar_{1}^{2}\right)(\theta)\right)_{\omega}^{\Psi} \mathcal{R}_{0}^{\lambda}\left(\hbar_{2}^{2}\right)(\theta)\right)_{\omega}^{\Psi} \mathcal{R}_{0}^{\kappa}\left(f_{1} f_{2}\right)(\theta)} .
\end{aligned}
$$

It follows that

$$
\begin{aligned}
& { }_{\omega}^{\Psi} \mathcal{R}_{0}^{\lambda}\left(g_{1} g_{2}\right)(\theta){ }_{\omega}^{\Psi} \mathcal{R}_{0}^{\kappa}\left(\hbar_{1}^{2}\right)(\theta)_{\omega}^{\Psi} \mathcal{R}_{0}^{\lambda}\left(\hbar_{2}^{2}\right)(\theta){ }_{\omega}^{\Psi} \mathcal{R}_{0}^{\kappa}\left(f_{1} f_{2}\right)(\theta) \\
& \leq \frac{1}{4}\left({ }_{\omega}^{\Psi} \mathcal{R}_{0}^{\lambda}\left(g_{1} \hbar_{2}\right)(\theta){ }_{\omega}^{\Psi} \mathcal{R}_{0}^{\kappa}\left(f_{1} \hbar_{1}\right)(\theta)+{ }_{\omega}^{\Psi} \mathcal{R}_{0}^{\lambda}\left(g_{2} \hbar_{2}\right)(\theta){ }_{\omega}^{\Psi} \mathcal{R}_{0}^{\kappa}\left(f_{2} \hbar_{1}\right)(\theta)\right)^{2},
\end{aligned}
$$

which completes the desired assertion (3.8).

Corollary 3.2 Let the functions $\hbar_{1}$ and $\hbar_{2}$ be positive and integrable on $[0, \infty)$ satisfying hypothesis $\left(H_{2}\right)$ defined by (3.7), and assume that the function $\Psi$ is increasing, positive, and monotone on $[0, \infty[$ and having continuous derivative on $[0, \infty[$ with $\Psi(0)=0$. Then, for $\kappa, \lambda>0$ and $\theta>0$, the following weighted fractional integral inequality holds:

$$
\frac{\omega^{-2}(\theta)^{\Psi} \mathcal{R}_{0}^{\kappa}(\omega)(\theta)^{\Psi} \mathcal{R}_{0}^{\lambda}(\omega)(\theta)_{\omega}^{\Psi} \mathcal{R}_{0}^{\kappa}\left(\hbar_{1}^{2}\right)(\theta)_{\omega}^{\Psi} \mathcal{R}_{0}^{\lambda}\left(\hbar_{2}^{2}\right)(\theta)}{\left({ }_{\omega}^{\Psi} \mathcal{R}_{0}^{\kappa}\left(\hbar_{1}\right)(\theta)_{\omega}^{\Psi} \mathcal{R}_{0}^{\lambda}\left(\hbar_{2}\right)(\theta)\right)^{2}} \leq \frac{1}{4}\left(\sqrt{\frac{p_{1} q_{1}}{P_{1} Q_{1}}}+\sqrt{\frac{P_{1} Q_{1}}{p_{1} q_{1}}}\right)^{2},
$$

where

$$
{ }^{\Psi} \mathcal{R}_{0}^{\kappa}(\omega)(\theta)=\frac{1}{\Gamma(\kappa)} \int_{0}^{\theta}(\Psi(\theta)-\Psi(\vartheta))^{\kappa-1} \Psi^{\prime}(\vartheta) \omega(\vartheta) d \vartheta .
$$

Lemma 3.3 Suppose that all the conditions of Lemma 3.1 hold, and assume that the function $\Psi$ is increasing, positive, and monotone on $[0, \infty[$ and having continuous derivative on $[0, \infty[$ with $\Psi(0)=0$. Then, for $\kappa, \lambda>0$ and $\theta>0$, the following weighted fractional integral inequality holds:

$$
{ }_{\omega}^{\Psi} \mathcal{R}_{0}^{\kappa}\left(\hbar_{1}^{2}\right)(\theta){ }_{\omega}^{\Psi} \mathcal{R}_{0}^{\lambda}\left(\hbar_{2}^{2}\right)(\theta) \leq{ }_{\omega}^{\Psi} \mathcal{R}_{0}^{\kappa}\left(\frac{f_{2} \hbar_{1} \hbar_{2}}{g_{1}}\right)(\theta){ }_{\omega}^{\Psi} \mathcal{R}_{0}^{\lambda}\left(\frac{g_{2} \hbar_{1} \hbar_{2}}{f_{1}}\right)(\theta) .
$$

Proof From the hypothesis given by (3.1), we have

$$
\begin{aligned}
& \frac{\omega^{-1}(\theta)}{\Gamma(\kappa)} \int_{0}^{\vartheta}(\Psi(\theta)-\Psi(\vartheta))^{\kappa-1} \omega(\vartheta) \Psi^{\prime}(\vartheta) \hbar_{1}^{2}(\vartheta) d \vartheta \\
& \quad \leq \frac{\omega^{-1}(\theta)}{\Gamma(\kappa)} \int_{0}^{\theta}(\Psi(\theta)-\Psi(\vartheta))^{\kappa-1} \omega(\vartheta) \Psi^{\prime}(\vartheta) \frac{f_{2}(\vartheta)}{g_{1}(\vartheta)} \hbar_{1}(\vartheta) \hbar_{2}(\vartheta) d \vartheta
\end{aligned}
$$


which in view of (2.9) yields

$$
{ }_{\omega}^{\Psi} \mathcal{R}_{0}^{\kappa}\left(\hbar_{1}^{2}\right)(\theta) \leq{ }_{\omega}^{\Psi} \mathcal{R}_{0}^{\kappa}\left(\frac{f_{2} \hbar_{1} \hbar_{2}}{g_{1}}\right)(\theta)
$$

Similarly, one can obtain

$$
{ }_{\omega}^{\Psi} \mathcal{R}_{0}^{\lambda}\left(\hbar_{2}^{2}\right)(\theta) \leq{ }_{\omega}^{\Psi} \mathcal{R}_{0}^{\lambda}\left(\frac{g_{2} \hbar_{1} \hbar_{2}}{f_{1}}\right)(\theta)
$$

Hence, the product of (3.14) and (3.15) gives the desired assertion (3.13).

Corollary 3.3 Let the functions $\hbar_{1}$ and $\hbar_{2}$ be positive and integrable on $[0, \infty)$ satisfying hypothesis $\left(\mathrm{H}_{2}\right)$ defined by (3.7), and assume that the function $\Psi$ is increasing, positive, and monotone on $\left[0, \infty\left[\right.\right.$ and its derivative $\Psi^{\prime}$ is continuous on $[0, \infty[$ with $\Psi(0)=0$. Then, for $\kappa, \lambda>0$ and $\theta>0$, the following weighted fractional integral inequality holds:

$$
\frac{{ }_{\omega}^{\Psi} \mathcal{R}_{0}^{\kappa}\left(\hbar_{1}^{2}\right)(\theta)_{\omega}^{\Psi} \mathcal{R}_{0}^{\lambda}\left(\hbar_{2}^{2}\right)(\theta)}{{ }_{\omega}^{\Psi} \mathcal{R}_{0}^{\kappa}\left(\hbar_{1} \hbar_{2}\right)(\theta)_{\omega}^{\Psi} \mathcal{R}_{0}^{\lambda}\left(\hbar_{1} \hbar_{2}\right)(\theta)} \leq \frac{P_{1} Q_{1}}{p_{1} q_{1}}
$$

\section{Chebyshev type weighted fractional integral inequalities}

In this section, we present Chebyshev type weighted fractional integral inequalities by using the Pólya-Szegö integral inequality given by Lemma 3.1 by employing weighted fractional integral (2.9).

Theorem 4.1 Let the functions $\hbar_{1}$ and $\hbar_{2}$ be positive and integrable on $[0, \infty)$, and assume that the function $\Psi$ is increasing, positive, and monotone on $\left[0, \infty\left[\right.\right.$ and $\Psi^{\prime}$ is continuous on $\left[0, \infty\left[\right.\right.$ with $\Psi(0)=0$. Suppose that $f_{1}, f_{2}, g_{1}$, and $f_{2}$ are four positive and integrable functions on $[0, \infty)$ satisfying hypothesis $\left(H_{1}\right)$ defined by (3.1). Then, for $\kappa, \lambda>0$ and $\theta>0$, the following weighted fractional integral inequality holds:

$$
\begin{aligned}
& \mid \omega^{-1}(\theta)^{\Psi} \mathcal{R}_{0}^{\kappa}(\omega)(\theta){ }_{\omega}^{\Psi} \mathcal{R}_{0}^{\kappa}\left(\hbar_{1} \hbar_{2}\right)(\theta)+\omega^{-1}(\theta)^{\Psi} \mathcal{R}_{0}^{\lambda}(\omega)(\theta){ }_{\omega}^{\Psi} \mathcal{R}_{0}^{\lambda}\left(\hbar_{1} \hbar_{2}\right)(\theta) \\
& \quad-{ }_{\omega}^{\Psi} \mathcal{R}_{0}^{\kappa}\left(\hbar_{1}\right)(\theta)_{\omega}^{\Psi} \mathcal{R}_{0}^{\lambda}\left(\hbar_{2}\right)(\theta)-{ }_{\omega}^{\Psi} \mathcal{R}_{0}^{\kappa}\left(\hbar_{2}\right)(\theta){ }_{\omega}^{\Psi} \mathcal{R}_{0}^{\lambda}\left(\hbar_{1}\right)(\theta) \mid \\
& \leq\left|F_{1}\left(\hbar_{1}, f_{1}, f_{2}\right)(\theta)+F_{2}\left(\hbar_{1}, f_{1}, f_{2}\right)(\theta)\right|^{\frac{1}{2}} \\
& \quad \times\left|F_{1}\left(\hbar_{2}, g_{1}, g_{2}\right)(\theta)+F_{2}\left(\hbar_{1}, g_{1}, g_{2}\right)(\theta)\right|^{\frac{1}{2}}
\end{aligned}
$$

where

$$
F_{1}\left(\hbar_{1}, f_{1}, f_{2}\right)(\theta)=\frac{\omega^{-1}(\theta)^{\Psi} \mathcal{R}_{0}^{\lambda}(\omega)(\theta)\left({ }_{\omega}^{\Psi} \mathcal{R}_{0}^{\kappa}\left\{\left(f_{1}+f_{2}\right) \hbar_{1}\right\}\right)^{2}}{4_{\omega}^{\Psi} \mathcal{R}_{0}^{\kappa}\left(f_{1} f_{2}\right)(\theta)}-{ }_{\omega}^{\Psi} \mathcal{R}_{0}^{\kappa}\left(\hbar_{1}\right)(\theta){ }_{\omega}^{\Psi} \mathcal{R}_{0}^{\lambda}\left(\hbar_{1}\right)(\theta)
$$

and

$$
F_{2}\left(\hbar_{1}, f_{1}, f_{2}\right)(\theta)=\frac{\omega^{-1}(\theta)^{\Psi} \mathcal{R}_{0}^{\kappa}(\omega)(\theta)\left({ }_{\omega}^{\Psi} \mathcal{R}_{0}^{\lambda}\left\{\left(f_{1}+f_{2}\right) \hbar_{1}\right\}\right)^{2}}{4_{\omega}^{\Psi} \mathcal{R}_{0}^{\lambda}\left(f_{1} f_{2}\right)(\theta)}-{ }_{\omega}^{\Psi} \mathcal{R}_{0}^{\kappa}\left(\hbar_{1}\right)(\theta)_{\omega}^{\Psi} \mathcal{R}_{0}^{\lambda}\left(\hbar_{1}\right)(\theta) .
$$


Proof By the given hypothesis both the functions $\hbar_{1}$ and $\hbar_{2}$ are positive and integrable functions on $[0, \infty)$. Therefore, for $\vartheta, \zeta \in(0, \theta)$ with $\theta>0$, we define $\mathcal{A}(\vartheta, \zeta)$ by

$$
\begin{aligned}
\mathcal{A}(\vartheta, \zeta) & =\left(\hbar_{1}(\vartheta)-\hbar_{1}(\zeta)\right)\left(\hbar_{2}(\vartheta)-\hbar_{2}(\zeta)\right) \\
& =\hbar_{1}(\vartheta) \hbar_{2}(\vartheta)+\hbar_{1}(\zeta) \hbar_{2}(\zeta)-\hbar_{1}(\vartheta) \hbar_{2}(\zeta)-\hbar_{1}(\zeta) \hbar_{2}(\vartheta) .
\end{aligned}
$$

Multiplying (4.2) by $\frac{\omega^{-2}(\theta)}{\Gamma(\kappa) \Gamma(\lambda)}(\Psi(\theta)-\Psi(\vartheta))^{\kappa-1}(\Psi(\theta)-\Psi(\zeta))^{\lambda-1} \omega(\vartheta) \omega(\zeta) \Psi^{\prime}(\vartheta) \Psi^{\prime}(\zeta)$ and double integrating the resultant identity with respect to $\vartheta$ and $\zeta$ over $(0, \theta)$, and then using (2.9), we obtain

$$
\begin{aligned}
& \frac{\omega^{-2}(\theta)}{\Gamma(\kappa) \Gamma(\lambda)} \int_{0}^{\theta} \int_{0}^{\theta}(\Psi(\theta)-\Psi(\vartheta))^{\kappa-1}(\Psi(\theta)-\Psi(\zeta))^{\lambda-1} \\
& \quad \times \omega(\vartheta) \omega(\zeta) \Psi^{\prime}(\vartheta) \Psi^{\prime}(\zeta) \mathcal{A}(\vartheta, \zeta) d \vartheta d \zeta \\
& =\omega^{-1}(\theta)^{\Psi} \mathcal{R}_{0}^{\kappa}(\omega)(\theta){ }_{\omega}^{\Psi} \mathcal{R}_{0}^{\kappa}\left(\hbar_{1} \hbar_{2}\right)(\theta)+\omega^{-1}(\theta){ }^{\Psi} \mathcal{R}_{0}^{\lambda}(\omega)(\theta){ }_{\omega}^{\Psi} \mathcal{R}_{0}^{\kappa}\left(\hbar_{1} \hbar_{2}\right)(\theta) \\
& \quad-{ }_{\omega}^{\Psi} \mathcal{R}_{0}^{\kappa}\left(\hbar_{1}\right)(\theta){ }_{\omega}^{\Psi} \mathcal{R}_{0}^{\lambda}\left(\hbar_{2}\right)(\theta)-{ }_{\omega}^{\Psi} \mathcal{R}_{0}^{\lambda}\left(\hbar_{1}\right)(\theta){ }_{\omega}^{\Psi} \mathcal{R}_{0}^{\kappa}\left(\hbar_{2}\right)(\theta) .
\end{aligned}
$$

By applying the Cauchy-Schwarz inequality for double integrals, we have

$$
\begin{aligned}
\mid \frac{\omega^{-2}(\theta)}{\Gamma(\kappa)} \int_{0}^{\theta} \int_{0}^{\theta}(\Psi(\theta)-\Psi(\vartheta))^{\kappa-1}(\Psi(\theta)-\Psi(\zeta))^{\lambda-1} \omega(\vartheta) \omega(\zeta) \\
\quad \times \Psi^{\prime}(\vartheta) \Psi^{\prime}(\zeta) \mathcal{A}(\vartheta, \zeta) d \vartheta d \zeta \mid \\
\leq\left[\frac{\omega^{-2}(\theta)}{\Gamma(\kappa) \Gamma(\lambda)} \int_{0}^{\theta} \int_{0}^{\theta}(\Psi(\theta)-\Psi(\vartheta))^{\kappa-1}(\Psi(\theta)-\Psi(\zeta))^{\lambda-1} \omega(\vartheta) \omega(\zeta)\right. \\
\quad \times \Psi^{\prime}(\vartheta) \Psi^{\prime}(\zeta) \hbar_{1}^{2}(\vartheta) d \vartheta d \zeta \\
\quad+\frac{\omega^{-2}(\theta)}{\Gamma(\kappa) \Gamma(\lambda)} \int_{0}^{\theta} \int_{0}^{\theta}(\Psi(\theta)-\Psi(\vartheta))^{\kappa-1}(\Psi(\theta)-\Psi(\zeta))^{\lambda-1} \omega(\vartheta) \omega(\zeta) \\
\quad \times \Psi^{\prime}(\vartheta) \Psi^{\prime}(\zeta) \hbar_{1}^{2}(\zeta) d \vartheta d \zeta \\
\quad-2 \frac{\omega^{-2}(\theta)}{\Gamma(\kappa) \Gamma(\lambda)} \int_{0}^{\theta} \int_{0}^{\theta}(\Psi(\theta)-\Psi(\vartheta))^{\kappa-1}(\Psi(\theta)-\Psi(\zeta))^{\lambda-1} \omega(\vartheta) \omega(\zeta) \\
\left.\quad \times \Psi^{\prime}(\vartheta) \Psi^{\prime}(\zeta) \hbar_{1}(\vartheta) \hbar_{1}(\zeta) d \vartheta d \zeta\right]^{\frac{1}{2}} \\
\quad \times\left[\frac{\omega^{-2}(\theta)}{\Gamma(\kappa) \Gamma(\lambda)} \int_{0}^{\theta} \int_{0}^{\theta}(\Psi(\theta)-\Psi(\vartheta))^{\kappa-1}(\Psi(\theta)-\Psi(\zeta))^{\lambda-1} \omega(\vartheta) \omega(\zeta)\right. \\
\quad \times \Psi^{\prime}(\vartheta) \Psi^{\prime}(\zeta) \hbar_{2}^{2}(\vartheta) d \vartheta d \zeta \\
\quad+\frac{\omega^{-2}(\theta)}{\Gamma(\kappa) \Gamma(\lambda)} \int_{0}^{\theta} \int_{0}^{\theta}(\Psi(\theta)-\Psi(\vartheta))^{\kappa-1}(\Psi(\theta)-\Psi(\zeta))^{\lambda-1} \omega(\vartheta) \omega(\zeta) \\
\times \Psi^{\prime}(\vartheta) \Psi^{\prime}(\zeta) \hbar_{2}^{2}(\zeta) d \vartheta d \zeta \\
\quad-2 \frac{\omega^{-2}(\theta)}{\Gamma(\kappa) \Gamma(\lambda)} \int_{0}^{\theta} \int_{0}^{\theta}(\Psi(\theta)-\Psi(\vartheta))^{\kappa-1}(\Psi(\theta)-\Psi(\zeta))^{\lambda-1} \omega(\vartheta) \omega(\zeta) \\
\left.\times \Psi^{\prime}(\vartheta) \Psi^{\prime}(\zeta) \hbar_{2}(\vartheta) \hbar_{2}(\zeta) d \vartheta d \zeta\right]^{\frac{1}{2}} \cdot \\
\quad
\end{aligned}
$$


In view of (2.9) and (3.12), we get

$$
\begin{aligned}
& \mid \frac{\omega^{-2}(\theta)}{\Gamma(\kappa) \Gamma(\lambda)} \int_{0}^{\theta} \int_{0}^{\theta}(\Psi(\theta)-\Psi(\vartheta))^{\kappa-1}(\Psi(\theta)-\Psi(\zeta))^{\lambda-1} \omega(\vartheta) \omega(\zeta) \\
& \quad \times \Psi^{\prime}(\vartheta) \Psi^{\prime}(\zeta) \mathcal{A}(\vartheta, \zeta) d \vartheta d \zeta \mid \\
& \leq\left[\omega^{-1}(\theta)^{\Psi} \mathcal{R}_{0}^{\kappa}(\omega)(\theta)_{\omega}^{\Psi} \mathcal{R}_{0}^{\lambda}\left(\hbar_{1}^{2}\right)(\theta)+\omega^{-1}(\theta)^{\Psi} \mathcal{R}_{0}^{\lambda}(\omega)(\theta)_{\omega}^{\Psi} \mathcal{R}_{0}^{\kappa}\left(\hbar_{1}^{2}\right)(\theta)\right. \\
& \left.\quad-2_{\omega}^{\Psi} \mathcal{R}_{0}^{\kappa}\left(\hbar_{1}\right)(\theta)_{\omega}^{\Psi} \mathcal{R}_{0}^{\lambda}\left(\hbar_{1}\right)(\theta)\right]^{\frac{1}{2}} \\
& \quad \times\left[\omega^{-1}(\theta)^{\Psi} \mathcal{R}_{0}^{\kappa}(\omega)(\theta)_{\omega}^{\Psi} \mathcal{R}_{0}^{\lambda}\left(\hbar_{2}^{2}\right)(\theta)+\omega^{-1}(\theta)^{\Psi} \mathcal{R}_{0}^{\lambda}(\omega)(\theta)_{\omega}^{\Psi} \mathcal{R}_{0}^{\kappa}\left(\hbar_{2}^{2}\right)(\theta)\right. \\
& \left.\quad-2_{\omega}^{\Psi} \mathcal{R}_{0}^{\kappa}\left(\hbar_{2}\right)(\theta)_{\omega}^{\Psi} \mathcal{R}_{0}^{\lambda}\left(\hbar_{2}\right)(\theta)\right]^{\frac{1}{2}} .
\end{aligned}
$$

By applying Lemma 3.1 for $g_{1}(\theta)=g_{2}(\theta)=\hbar_{2}(\theta)=1$, we get

$$
\omega^{-1}(\theta){ }^{\Psi} \mathcal{R}_{0}^{\lambda}(\omega)(\theta)_{\omega}^{\Psi} \mathcal{R}_{0}^{\kappa}\left(\hbar_{1}^{2}\right)(\theta) \leq \frac{\omega^{-1}(\theta){ }^{\Psi} \mathcal{R}_{0}^{\lambda}(\omega)(\theta)\left({ }_{\omega}^{\Psi} \mathcal{R}_{0}^{\kappa}\left\{\left(f_{1}+f_{2}\right) \hbar_{1}\right\}\right)^{2}}{4_{\omega}^{\Psi} \mathcal{R}_{0}^{\kappa}\left(f_{1} f_{2}\right)(\theta)}
$$

It follows that

$$
\begin{aligned}
& \omega^{-1}(\theta)^{\Psi} \mathcal{R}_{0}^{\lambda}(\omega)(\theta){ }_{\omega}^{\Psi} \mathcal{R}_{0}^{\kappa}\left(\hbar_{1}^{2}\right)(\theta)-{ }_{\omega}^{\Psi} \mathcal{R}_{0}^{\kappa}\left(\hbar_{1}\right)(\theta){ }_{\omega}^{\Psi} \mathcal{R}_{0}^{\lambda}\left(\hbar_{1}\right)(\theta) \\
& \quad \leq \frac{\omega^{-1}(\theta)^{\Psi} \mathcal{R}_{0}^{\lambda}(\omega)(\theta)}{4} \frac{\left({ }_{\omega}^{\Psi} \mathcal{R}_{0}^{\kappa}\left\{\left(f_{1}+f_{2}\right) \hbar_{1}\right\}\right)^{2}}{{ }_{\omega}^{\Psi} \mathcal{R}_{0}^{\kappa}\left(f_{1} f_{2}\right)(\theta)}-{ }_{\omega}^{\Psi} \mathcal{R}_{0}^{\kappa}\left(\hbar_{1}\right)(\theta){ }_{\omega}^{\Psi} \mathcal{R}_{0}^{\lambda}\left(\hbar_{1}\right)(\theta) \\
& \quad=F_{1}\left(\hbar_{1}, f_{1}, f_{2}\right)(\theta) .
\end{aligned}
$$

Similarly, one can get

$$
\begin{aligned}
& \omega^{-1}(\theta)^{\Psi} \mathcal{R}_{0}^{\kappa}(\omega)(\theta)_{\omega}^{\Psi} \mathcal{R}_{0}^{\lambda}\left(\hbar_{1}^{2}\right)(\theta)-{ }_{\omega}^{\Psi} \mathcal{R}_{0}^{\kappa}\left(\hbar_{1}\right)(\theta)_{\omega}^{\Psi} \mathcal{R}_{0}^{\lambda}\left(\hbar_{1}\right)(\theta) \\
& \quad \leq \frac{\omega^{-1}(\theta)^{\Psi} \mathcal{R}_{0}^{\kappa}(\omega)(\theta)}{4} \frac{\left({ }_{\omega}^{\Psi} \mathcal{R}_{0}^{\kappa}\left\{\left(f_{1}+f_{2}\right) \hbar_{1}\right\}\right)^{2}}{{ }_{\omega}^{\Psi} \mathcal{R}_{0}^{\lambda}\left(f_{1} f_{2}\right)(\theta)}-{ }_{\omega}^{\Psi} \mathcal{R}_{0}^{\kappa}\left(\hbar_{1}\right)(\theta){ }_{\omega}^{\Psi} \mathcal{R}_{0}^{\lambda}\left(\hbar_{1}\right)(\theta) \\
& \quad=F_{2}\left(\hbar_{1}, f_{1}, f_{2}\right)(\theta) .
\end{aligned}
$$

Again applying Lemma 3.1 for $f_{1}(\theta)=f_{2}(\theta)=\hbar_{1}(\theta)=1$, we get

$$
\begin{aligned}
& \omega^{-1}(\theta)^{\Psi} \mathcal{R}_{0}^{\lambda}(\omega)(\theta){ }_{\omega}^{\Psi} \mathcal{R}_{0}^{\kappa}\left(\hbar_{2}^{2}\right)(\theta)-{ }_{\omega}^{\Psi} \mathcal{R}_{0}^{\kappa}\left(\hbar_{2}\right)(\theta){ }_{\omega}^{\Psi} \mathcal{R}_{0}^{\lambda}\left(\hbar_{2}\right)(\theta) \\
& \quad \leq \frac{\omega^{-1}(\theta)^{\Psi} \mathcal{R}_{0}^{\lambda}(\omega)(\theta)}{4} \frac{\left({ }_{\omega}^{\Psi} \mathcal{R}_{0}^{\kappa}\left\{\left(g_{1}+g_{2}\right) \hbar_{2}\right\}\right)^{2}}{{ }_{\omega}^{\Psi} \mathcal{R}_{0}^{\kappa}\left(g_{1} g_{2}\right)(\theta)}-{ }_{\omega}^{\Psi} \mathcal{R}_{0}^{\kappa}\left(\hbar_{2}\right)(\theta){ }_{\omega}^{\Psi} \mathcal{R}_{0}^{\lambda}\left(\hbar_{2}\right)(\theta) \\
& \quad=F_{1}\left(\hbar_{2}, f_{1}, f_{2}\right)(\theta)
\end{aligned}
$$

and

$$
\begin{gathered}
\omega^{-1}(\theta)^{\Psi} \mathcal{R}_{0}^{\kappa}(\omega)(\theta)_{\omega}^{\Psi} \mathcal{R}_{0}^{\lambda}\left(\hbar_{2}^{2}\right)(\theta)-{ }_{\omega}^{\Psi} \mathcal{R}_{0}^{\kappa}\left(\hbar_{2}\right)(\theta)_{\omega}^{\Psi} \mathcal{R}_{0}^{\lambda}\left(\hbar_{2}\right)(\theta) \\
\leq \frac{\omega^{-1}(\theta)^{\Psi} \mathcal{R}_{0}^{\kappa}(\omega)(\theta)}{4} \frac{\left({ }_{\omega}^{\Psi} \mathcal{R}_{0}^{\kappa}\left\{\left(g_{1}+g_{2}\right) \hbar_{2}\right\}\right)^{2}}{{ }_{\omega}^{\Psi} \mathcal{R}_{0}^{\lambda}\left(g_{1} g_{2}\right)(\theta)}
\end{gathered}
$$




$$
\begin{aligned}
& -{ }_{\omega}^{\Psi} \mathcal{R}_{0}^{\kappa}\left(\hbar_{2}\right)(\theta){ }_{\omega}^{\Psi} \mathcal{R}_{0}^{\lambda}\left(\hbar_{2}\right)(\theta) \\
= & F_{2}\left(\hbar_{2}, f_{1}, f_{2}\right)(\theta) .
\end{aligned}
$$

Thus, by considering (4.3) to (4.8), we arrive at the desired assertion (4.1) of Theorem 4.1.

Theorem 4.2 Suppose that all the conditions of Theorem 4.1 are satisfied. Then, for $\kappa>0$ and $\theta>0$, the following weighted fractional integral inequality holds:

$$
\begin{aligned}
& \left|\omega^{-1}(\theta)^{\Psi} \mathcal{R}_{0}^{\kappa}(\omega)(\theta)_{\omega}^{\Psi} \mathcal{R}_{0}^{\kappa}\left(\hbar_{1} \hbar_{2}\right)(\theta)-{ }_{\omega}^{\Psi} \mathcal{R}_{0}^{\kappa}\left(\hbar_{1}\right)(\theta)_{\omega}^{\Psi} \mathcal{R}_{0}^{\kappa}\left(\hbar_{2}\right)(\theta)\right| \\
& \quad \leq\left|F\left(\hbar_{1}, f_{1}, f_{2}\right)(\theta) F\left(\hbar_{2}, g_{1}, g_{2}\right)(\theta)\right|^{\frac{1}{2}},
\end{aligned}
$$

where

$$
F\left(\hbar_{1}, f_{1}, f_{2}\right)(\theta)=\frac{\omega^{-1}(\theta)^{\Psi} \mathcal{R}_{0}^{\kappa}(\omega)(\theta)}{4} \frac{\left({ }_{\omega}^{\Psi} \mathcal{R}_{0}^{\kappa}\left\{\left(f_{1}+f_{2}\right) \hbar_{1}\right\}\right)^{2}}{{ }_{\omega}^{\Psi} \mathcal{R}_{0}^{\kappa}\left(f_{1} f_{2}\right)(\theta)}-\left({ }_{\omega}^{\Psi} \mathcal{R}_{0}^{\kappa}\left(\hbar_{1}\right)(\theta)\right)^{2} .
$$

Proof Applying Theorem 4.1 for $\kappa=\lambda$, we get the desired assertion (4.9) of Theorem 4.2.

Remark 4.1 If we take $f_{1}=p_{1}, f_{2}=P_{1}, g_{1}=q_{1}$, and $g_{2}=Q_{1}$, then we have

$$
F\left(\hbar_{1}, p_{1}, P_{1}\right)(\theta)=\frac{\left(P_{1}-p_{1}\right)^{2}}{4 P_{1} p_{1}}\left({ }^{\Psi} \mathcal{R}_{0}^{\kappa, \tau}\left(\hbar_{1}\right)(\theta)\right)^{2}
$$

and

$$
F\left(\hbar_{2}, q_{1}, Q_{1}\right)(\theta)=\frac{\left(Q_{1}-q_{1}\right)^{2}}{4 Q_{1} q_{1}}\left({ }^{\Psi} \mathcal{R}_{0}^{\kappa, \tau}\left(\hbar_{2}\right)(\theta)\right)^{2} .
$$

Corollary 4.1 Let the functions $\hbar_{1}$ and $\hbar_{2}$ be positive and integrable on $[0, \infty)$ and satisfy hypothesis $\left(H_{2}\right)$ given by (3.7). Then, for $\kappa>0$ and $\theta>0$, the following tempered fractional integral inequality holds:

$$
\begin{aligned}
& \left|\omega^{-1}(\theta)^{\Psi} \mathcal{R}_{0}^{\kappa}(\omega)(\theta)_{\omega}^{\Psi} \mathcal{R}_{0}^{\kappa}\left(\hbar_{1} \hbar_{2}\right)(\theta)-{ }_{\omega}^{\Psi} \mathcal{R}_{0}^{\kappa}\left(\hbar_{1}\right)(\theta)_{\omega}^{\Psi} \mathcal{R}_{0}^{\kappa}\left(\hbar_{2}\right)(\theta)\right| \\
& \quad \leq \frac{\left(P_{1}-p_{1}\right)\left(Q_{1}-q_{1}\right)}{4 \sqrt{p_{1} P_{1} q_{1} Q_{1}}}{ }_{\omega}^{\Psi} \mathcal{R}_{0}^{\kappa}\left(\hbar_{1}\right)(\theta)_{\omega}^{\Psi} \mathcal{R}_{0}^{\kappa}\left(\hbar_{2}\right)(\theta) .
\end{aligned}
$$

\section{Special cases}

The following new Pólya-Szegö and Chebyshev type inequalities for one-sided weighted Riemann-Liouville fractional integral (2.5) can be easily derived.

Lemma 5.1 Let the functions $\hbar_{1}$ and $\hbar_{2}$ be positive and integrable on $[0, \infty)$. Suppose that $f_{1}, f_{2}, g_{1}$, and $f_{2}$ are four positive and integrable functions on $[0, \infty)$ satisfying hypothesis $\left(H_{1}\right)$ defined by (3.1). Then, for $\kappa>0$ and $\theta>0$, the following weighted fractional integral inequality holds:

$$
\frac{{ }_{\omega} \mathcal{R}_{0}^{\kappa}\left(g_{1} g_{2} \hbar_{1}^{2}\right)(\theta)_{\omega} \mathcal{R}_{0}^{\kappa}\left(f_{1} f_{2} \hbar_{2}^{2}\right)(\theta)}{\left({ }_{\omega} \mathcal{R}_{0}^{\kappa}\left\{\left(f_{1} g_{1}+f_{2} g_{2}\right) \hbar_{1} \hbar_{2}\right\}(\theta)\right)^{2}} \leq \frac{1}{4} .
$$


Proof Applying Lemma 3.1 for $\Psi(\theta)=\theta$, we get Lemma 5.1.

Lemma 5.2 Let all the conditions of Lemma 5.1 be satisfied. Then, for $\kappa, \lambda>0$ and $\theta>0$, the following weighted fractional integral inequality holds:

$$
\frac{{ }_{\omega} \mathcal{R}_{0}^{\kappa}\left(\hbar_{1}^{2}\right)(\theta)_{\omega} \mathcal{R}_{0}^{\lambda}\left(\hbar_{2}^{2}\right)(\theta)_{\omega} \mathcal{R}_{0}^{\kappa}\left(f_{1} f_{2}\right)(\theta)_{\omega} \mathcal{R}_{0}^{\lambda}\left(g_{1} g_{2}\right)(\theta)}{\left.{ }_{\omega} \mathcal{R}_{0}^{\kappa}\left(f_{1} \hbar_{1}\right)(\theta)_{\omega} \mathcal{R}_{0}^{\lambda}\left(g_{1} \hbar_{2}\right)(\theta)+{ }_{\omega} \mathcal{R}_{0}^{\kappa}\left(f_{2} \hbar_{1}\right)(\theta)_{\omega} \mathcal{R}_{0}^{\lambda}\left(g_{2} \hbar_{2}\right)(\theta)\right)^{2}} \leq \frac{1}{4}
$$

Proof Applying Lemma 3.2 for $\Psi(\theta)=\theta$, we get Lemma 5.2.

Similarly, one can derive the special case of Lemma 3.3. The following theorem represents the special case of Theorem 4.1 in terms of weighted classical fractional integral.

Theorem 5.1 Let the functions $\hbar_{1}$ and $\hbar_{2}$ be positive and integrable on $[0, \infty)$. Suppose that $f_{1}, f_{2}, g_{1}$, and $f_{2}$ are four positive and integrable functions on $[0, \infty)$ satisfying hypothesis $\left(H_{1}\right)$ defined by (3.1). Then, for $\kappa, \lambda>0$ and $\theta>0$, the following weighted fractional integral inequality holds:

$$
\begin{aligned}
& \mid \omega^{-1}(\theta) \mathcal{R}_{0}^{\kappa}(\omega)(\theta)_{\omega} \mathcal{R}_{0}^{\kappa}\left(\hbar_{1} \hbar_{2}\right)(\theta)+\omega^{-1}(\theta) \mathcal{R}_{0}^{\lambda}(\omega)(\theta)_{\omega} \mathcal{R}_{0}^{\lambda}\left(\hbar_{1} \hbar_{2}\right)(\theta) \\
& \quad-{ }_{\omega} \mathcal{R}_{0}^{\kappa}\left(\hbar_{1}\right)(\theta){ }_{\omega} \mathcal{R}_{0}^{\lambda}\left(\hbar_{2}\right)(\theta)-{ }_{\omega} \mathcal{R}_{0}^{\kappa}\left(\hbar_{2}\right)(\theta){ }_{\omega} \mathcal{R}_{0}^{\lambda}\left(\hbar_{1}\right)(\theta) \mid \\
& \leq\left|F_{1}\left(\hbar_{1}, f_{1}, f_{2}\right)(\theta)+F_{2}\left(\hbar_{1}, f_{1}, f_{2}\right)(\theta)\right|^{\frac{1}{2}} \times\left|F_{1}\left(\hbar_{2}, g_{1}, g_{2}\right)(\theta)+F_{2}\left(\hbar_{1}, g_{1}, g_{2}\right)(\theta)\right|^{\frac{1}{2}},
\end{aligned}
$$

where

$$
F_{1}\left(\hbar_{1}, f_{1}, f_{2}\right)(\theta)=\frac{\omega^{-1}(\theta) \mathcal{R}_{0}^{\lambda}(\omega)(\theta)}{4} \frac{\left({ }_{\omega} \mathcal{R}_{0}^{\kappa}\left\{\left(f_{1}+f_{2}\right) \hbar_{1}\right\}\right)^{2}}{{ }_{\omega} \mathcal{R}_{0}^{\kappa}\left(f_{1} f_{2}\right)(\theta)}-{ }_{\omega} \mathcal{R}_{0}^{\kappa}\left(\hbar_{1}\right)(\theta){ }_{\omega} \mathcal{R}_{0}^{\lambda}\left(\hbar_{1}\right)(\theta)
$$

and

$$
F_{2}\left(\hbar_{1}, f_{1}, f_{2}\right)(\theta)=\frac{\omega^{-1}(\theta) \mathcal{R}_{0}^{\kappa}(\omega)(\theta)}{4} \frac{\left.{ }_{\omega} \mathcal{R}_{0}^{\lambda}\left\{\left(f_{1}+f_{2}\right) \hbar_{1}\right\}\right)^{2}}{{ }_{\omega} \mathcal{R}_{0}^{\lambda}\left(f_{1} f_{2}\right)(\theta)}-{ }_{\omega} \mathcal{R}_{0}^{\kappa}\left(\hbar_{1}\right)(\theta){ }_{\omega} \mathcal{R}_{0}^{\lambda}\left(\hbar_{1}\right)(\theta) .
$$

Proof By employing Theorem 4.1 for $\Psi(\theta)=\theta$, we get the desired Theorem 5.1.

By applying different choices given in Remark 2.1, some new inequalities can be obtained easily. Also, we can derive the particular cases of the main result by employing Remark 2.2.

\section{Applications}

Here, we define a way for constructing four bounded functions and then utilize them to present certain estimates of Chebyshev type weighted fractional integral inequalities of two unknown functions.

Suppose that $\hbar(\theta)$ is the unit function defined by

$$
\hbar(\theta)= \begin{cases}1, & \theta>0, \\ 0, & \theta \leq 0\end{cases}
$$


and let $\hbar_{a}(\theta)$ be the Heaviside unit step function defined by

$$
\hbar_{a}(\theta)= \begin{cases}1, & \theta>a, \\ 0, & \theta \leq a .\end{cases}
$$

Suppose that the function $f_{1}$ is a piecewise continuous function on $[0, X]$ defined by

$$
\begin{aligned}
f_{1}(x) & =p_{1_{1}}\left(\hbar_{0}(x)-\hbar_{x_{1}}(x)\right)+p_{1_{2}}\left(\hbar_{x_{1}}(x)-\hbar_{x_{2}}(x)\right)+\cdots+p_{1_{m+1}} \hbar_{x_{m}}(x) \\
& =p_{1_{1}} \hbar_{0}(x)+\left(p_{1_{2}}-p_{1_{1}}\right) \hbar_{x_{1}}(x)+\left(p_{1_{3}}-p_{1_{2}}\right) \hbar_{x_{2}}(x)+\cdots+\left(p_{1_{m+1}}-p_{1_{m}}\right) \hbar_{x_{m}}(x) \\
& =\sum_{i=0}^{m}\left(p_{1_{i+1}}-p_{1_{i}}\right) \hbar_{x_{i}}(x),
\end{aligned}
$$

where $p_{1_{0}}=0$ and $0=x_{0}<x_{1}<x_{2}<\cdots<x_{p}<x_{p+1}=X$. Similarly, we define

$$
\begin{aligned}
& f_{2}(x)=\sum_{i=0}^{m}\left(P_{1_{i+1}}-P_{1_{i}}\right) \hbar_{x_{i}}(x), \\
& g_{1}(x)=\sum_{i=0}^{m}\left(q_{1_{i+1}}-q_{1_{i}}\right) \hbar_{x_{i}}(x),
\end{aligned}
$$

and

$$
g_{2}(x)=\sum_{i=0}^{m}\left(Q_{1_{i+1}}-Q_{1_{i}}\right) \hbar_{x_{i}}(x),
$$

where $q_{1_{0}}=Q_{1_{0}}=P_{1_{0}}=0$. If there exists an integrable function $\hbar_{1}$ on $[0, X]$ satisfying hypothesis $\left(H_{1}\right)$, then we have $p_{1_{i+1}} \leq \hbar_{1}(x) \leq P_{1_{i+1}}$ for each $x \in\left(x_{i}, x_{i+1}\right], i=0,1,2, \ldots, m$.

Proposition 6.1 Let the functions $\hbar_{1}$ and $\hbar_{2}$ be positive and integrable on $[0, X]$. Assume that the functions $f_{1}, f_{2}, g_{1}$, and $g_{2}$ are defined by (6.1), (6.2), (6.3), and (6.4) respectively and satisfy hypothesis $\left(H_{1}\right)$ defined by (3.7). Then, for $\kappa>0$, the following inequality for weighted fractional integral holds:

$$
\begin{aligned}
& \left(\sum_{i=0}^{m} q_{1_{i+1}} Q_{1_{i+1} \omega} \mathcal{W}_{x_{i}, x_{i+1}}^{\kappa}\left(\hbar_{1}^{2}\right)(X)\right)^{2}\left(\sum_{i=0}^{m} p_{1_{i+1}} P_{1_{i+1}} \underset{\Psi}{\Psi} \mathcal{R}_{x_{i}, x_{i+1}}^{\kappa}\left(\hbar_{2}^{2}\right)(X)\right)^{2} \\
& \quad \leq \frac{1}{4} \sum_{i=0}^{p}\left(q_{1_{i+1}} Q_{1_{i+1}}+p_{1_{i+1}} P_{1_{i+1}}\right)_{\omega}^{\Psi} \mathcal{R}_{x_{i}, x_{i+1}}^{\kappa}\left(\hbar_{1} \hbar_{2}\right)(X) .
\end{aligned}
$$

Proof By applying Definition (2.10), we have

$$
\begin{aligned}
& { }_{\omega}^{\Psi} \mathcal{R}_{0, X}^{\kappa}\left(g_{1} g_{2} \hbar_{1}^{2}\right)(X)=\sum_{i=0}^{m} q_{1_{i+1}} Q_{1_{i+1} \omega} \mathcal{R}_{x_{i}, x_{i+1}}^{\kappa}\left(\hbar_{1}^{2}\right)(X), \\
& { }_{\omega}^{\Psi} \mathcal{R}_{0, X}^{\kappa}\left(f_{1} f_{2} \hbar_{2}^{2}\right)(X)=\sum_{i=0}^{m} p_{1_{i+1}} P_{1_{i+1} \omega} \mathcal{R}_{x_{i}, x_{i+1}}^{\kappa}\left(\hbar_{2}^{2}\right)(X),
\end{aligned}
$$


and

$$
{ }_{\omega}^{\Psi} \mathcal{R}_{0, X}^{\kappa}\left\{\left(f_{1} g_{1}+f_{2} g_{2}\right) \hbar_{1} \hbar_{2}\right\}(X)=\sum_{i=0}^{m}\left(p_{1_{i+1}} q_{1_{i+1}}+P_{1_{i+1}} Q_{1_{i+1}}\right)_{\omega}^{\Psi} \mathcal{R}_{x_{i}, x_{i+1}}^{\kappa}\left(\hbar_{1} \hbar_{2}\right)(X) .
$$

Hence, by applying Lemma 3.1, we get the desired assertion (6.5).

Proposition 6.2 Applying Proposition 6.1 for $\Psi(\theta)=\theta$, we get the following result in terms of weighted $R-L$ fractional integral:

$$
\begin{aligned}
& \left(\sum_{i=0}^{m} q_{1_{i+1}} Q_{1_{i+1} \omega} \mathcal{R}_{x_{i}, x_{i+1}}^{\kappa}\left(\hbar_{1}^{2}\right)(X)\right)^{2}\left(\sum_{i=0}^{m} p_{1_{i+1}} P_{1_{i+1} \omega} \mathcal{R}_{x_{i}, x_{i+1}}^{\kappa}\left(\hbar_{2}^{2}\right)(X)\right)^{2} \\
& \quad \leq \frac{1}{4} \sum_{i=0}^{m}\left(q_{1_{i+1}} Q_{1_{i+1}}+p_{1_{i+1}} P_{1_{i+1}}\right)_{\omega} \mathcal{R}_{x_{i}, x_{i+1}}^{\kappa}\left(\hbar_{1} \hbar_{2}\right)(X) .
\end{aligned}
$$

Proposition 6.3 Let the functions $\hbar_{1}$ and $\hbar_{2}$ be positive and integrable on [0,X]. Assume that the functions $f_{1}, f_{2}, g_{1}$, and $g_{2}$ are defined by (6.1), (6.2), (6.3), and (6.4) respectively and satisfy hypothesis $\left(H_{1}\right)$ defined by (3.7). Then, for $\kappa>0$, the following inequality for generalized fractional integral holds:

$$
\begin{gathered}
\left(\sum_{i=0}^{m} q_{1_{i+1}} Q_{1_{i+1}}^{\Psi} \mathcal{R}_{x_{i}, x_{i+1}}^{\kappa}\left(\hbar_{1}^{2}\right)(X)\right)^{2}\left(\sum_{i=0}^{m} p_{1_{i+1}} P_{1_{i+1}}^{\Psi} \mathcal{R}_{x_{i}, x_{i+1}}^{\kappa}\left(\hbar_{2}^{2}\right)(X)\right)^{2} \\
\leq \frac{1}{4} \sum_{i=0}^{p}\left(q_{1_{i+1}} Q_{1_{i+1}}+p_{1_{i+1}} P_{1_{i+1}}\right)^{\Psi} \mathcal{R}_{x_{i}, x_{i+1}}^{\kappa}\left(\hbar_{1} \hbar_{2}\right)(X) .
\end{gathered}
$$

Remark 6.1 By setting $\Psi(\theta)=\theta$ and $\omega(\theta)=1$ throughout the paper, we obtain the work of Ntouyas et al. [31].

\section{Concluding remarks}

In this present investigation, we presented some new weighted fractional Pólya-Szegö and Chebyshev type integral inequalities by employing weighted fractional integral recently proposed by Jarad et al. [14]. It is worth mentioning that these inequalities cover the integral inequalities for the well-known fractional integral operators discussed in Remark 2.2. In particular, if we take $\Psi(\theta)=\theta$ and $\omega(\theta)=1$, then the obtained inequalities reduce to the inequalities involving the R-L fractional integral established by Ntouyas et al. [31]. One can easily obtain Pólya-Szegö and Chebyshev type Hadamard fractional integral inequalities by applying $\Psi(\theta)=\ln \theta$ and $\omega(\theta)=\theta^{u}$. Also, one can easily derive the said Pólya-Szegö and Chebyshev type inequalities for other types of weighted fractional integrals such as Katugampola, generalized R-L, classical R-L, generalized conformable, and conformable fractional integrals by applying certain conditions on the function $\Psi$ given in Remark 2.1. 


\section{Competing interests}

The authors declare that they have no competing interests.

\section{Authors' contributions}

All the authors contributed equally and they read and approved the final manuscript for publication.

\section{Author details}

${ }^{1}$ Informetrics Research Group, Ton Duc Thang University, Ho Chi Minh City, Vietnam. ${ }^{2}$ Faculty of Mathematics and Statistics, Ton Duc Thang University, Ho Chi Minh City, Vietnam. ${ }^{3}$ Department of Mathematics, Shaheed Benazir Bhutto University, Sheringal, 18000, Upper Dir, Pakistan. ${ }^{4}$ Department of Mathematics, Cankaya University, Ankara 06790, Turkey. ${ }^{5}$ Institute of Space Sciences, Magurele-Bucharest 077125, Romania. ${ }^{6}$ Department of Medical Research, China Medical University, Taichung 40447, Taiwan. ${ }^{7}$ Department of Mathematics, University of Sargodha, Sargodha, Pakistan.

${ }^{8}$ Department of Mathematics, Riphah international university, Faisalabad Campus, Satyana road, Faisalabad, Pakistan.

\section{Publisher's Note}

Springer Nature remains neutral with regard to jurisdictional claims in published maps and institutional affiliations.

Received: 3 August 2020 Accepted: 21 October 2020 Published online: 06 November 2020

\section{References}

1. Abdeljawad, T., Mohammed, P.O., Kashuri, A.: New modified conformable fractional integral inequalities of Hermite-Hadamard type with applications. J. Funct. Spaces 2020, Article ID 4352357 (2020). https://doi.org/10.1155/2020/4352357

2. Abdeljawad, T., Rashid, S., Hammouch, Z, Chu, Y.M.: Some new local fractional inequalities associated with generalized ( $s, m)$-convex functions and applications. Adv. Differ. Equ. 2020, 406 (2020). https://doi.org/10.1186/s13662-020-02865-w

3. Abdeljawad, T., Rashid, S., Khan, H., Chu, Y.M.: On new fractional integral inequalities for p-convexity within interval-valued functions. Adv. Differ. Equ. 2020, 330 (2020). https://doi.org/10.1186/s13662-020-02782-y

4. Belarbi, S., Dahmani, Z:: On some new fractional integral inequalities. J. Inequal. Pure Appl. Math. 10(3), 1-12 (2009)

5. Chebyshev, P.L.: Sur les expressions approximatives des integrales definies par les autres prises entre les mêmes limites. Proc. Math. Soc. Charkov 2,93-98 (1882)

6. Dahmani, Z.: New inequalities in fractional integrals. Int. J. Nonlinear Sci. 9, 493-497 (2010)

7. Dahmani, Z.: About some integral inequalities using Riemann-Liouville integrals. Gen. Math. 20(4), 63-69 (2012)

8. Dahmani, Z., Tabharit, L.: On weighted Gruss type inequalities via fractional integration. J. Adv. Res. Pure Math. 2 $31-38(2010)$

9. Dragomir, S.S.: A generalization of Gruss' inequality in inner product spaces and applications. J. Math. Anal. Appl. 237(1), 74-82 (1999)

10. Dragomir, S.S., Diamond, N.T.: Integral inequalities of Grüss type via Pólya-Szegö and Shisha-Mond results. East Asian Math. J. 19(1), 27-39 (2003)

11. Grüss, G.: Uber, das Maximum des absoluten Betrages von $\frac{1}{b-a} \int_{a}^{b} \mathcal{U}(\varrho) \mathcal{V}(\varrho) d \varrho-\frac{1}{(b-a)^{2}} \int_{a}^{b} \mathcal{U}(\varrho) d \varrho \int_{a}^{b} \mathcal{V}(\varrho) d \varrho$. Math. Z. 39, 215-226 (1935)

12. Huang, C.J., Rahman, G., Nisar, K.S., Ghaffar, A., Qi, F.: Some Inequalities of Hermite-Hadamard type for k-fractional conformable integrals. Aust. J. Math. Anal. Appl. 16(1), 1-9 (2019)

13. Jarad, F., Abdeljawad, T., Alzabut, J.: Generalized fractional derivatives generated by a class of local proportional derivatives. Eur. Phys. J. Spec. Top. 226, 3457-3471 (2017). https://doi.org/10.1140/epjst/e2018-00021-7

14. Jarad, F., Abdeljawad, T., Shah, K.: On the weighted fractional operators of a function with respect to another function. Fractals (2020). https://doi.org/10.1142/S0218348X20400113

15. Jarad, F., Ugurlu, E., Abdeljawad, T., Baleanu, D.: On a new class of fractional operators. Adv. Differ. Equ. 2017, 247 (2017)

16. Kacar, E., Kacar, Z., Yildirim, H.: Integral inequalities for Riemann-Liouville fractional integrals of a function with respect to another function. Iran. J. Math. Sci. Inform. 13, 1-13 (2018)

17. Kalla, S.L., Rao, A: On Grüss type inequality for hypergeometric fractional integrals. Matematiche 66(1), 57-64 (2011)

18. Katugampola, U.N.: Approach to a generalized fractional integral. Appl. Math. Comput. 218, 860-865 (2011)

19. Khan, T.U., Khan, M.A.: Generalized conformable fractional integral operators. J. Comput. Appl. Math. 346, 378-389 (2019)

20. Kilbas, A.A., Sarivastava, H.M., Trujillo, J.J.: Theory and Application of Fractional Differential Equation. North-Holland Mathematics Studies. Elsevier, Amsterdam (2006)

21. Mohammed, P.O., Abdeljawad, T.: Modification of certain fractional integral inequalities for convex functions. Adv. Differ. Equ. 2020, 69 (2020). https://doi.org/10.1186/s13662-020-2541-2

22. Mohammed, P.O., Abdeljawad, T.: Integral inequalities for a fractional operator of a function with respect to another function with nonsingular kernel. Adv. Differ. Equ. 2020, 363 (2020). https://doi.org/10.1186/s13662-020-02825-4

23. Mohammed, P.O., Brevik, I.: A new version of the Hermite-Hadamard inequality for Riemann-Liouville fractional integrals. Symmetry 12(4), 610 (2020). https://doi.org/10.3390/sym12040610

24. Mohammed, P.O., Sarikaya, M.Z.: On generalized fractional integral inequalities for twice differentiable convex functions. J. Comput. Appl. Math. 2020, 372 (2020)

25. Niasr, K.S., Tassadiq, A., Rahman, G., Khan, A.: Some inequalities via fractional conformable integral operators. J. Inequal. Appl. 2019, 217 (2019). https://doi.org/10.1186/s13660-019-2170-z

26. Nisar, K.S., Qi, F., Rahman, G., Mubeen, S., Arshad, M.: Some inequalities involving the extended gamma function and the Kummer confluent hypergeometric k-function. J. Inequal. Appl. 2018, 135 (2018)

27. Nisar, K.S., Rahman, G., Choi, J., Mubeen, S., Arshad, M.: Certain Gronwall type inequalities associated with Riemann-Liouville k- and Hadamard k-fractional derivatives and their applications. East Asian Math. J. 34(3), 249-263 (2018) 
28. Nisar, K.S., Rahman, G., Khan, A.: Some new inequalities for generalized fractional conformable integral operators. Adv. Differ. Equ. 2019, 427 (2019). https://doi.org/10.1186/s13662-019-2362-3

29. Nisar, K.S., Rahman, G., Khan, A., Tassaddiq, A., Abouzaid, M.S.: Certain generalized fractional integral inequalities. AIMS Math. 5(2), 1588-1602 (2020). https://doi.org/10.3934/math.2020108

30. Nisar, K.S., Rahman, G., Mehrez, K.: Chebyshev type inequalities via generalized fractional conformable integrals. J. Inequal. Appl. 2019, 245 (2019). https://doi.org/10.1186/s13660-019-2197-1

31. Ntouyas, S.K., Agarwal, P., Tariboon, J.: On Pólya-Szegö and Chebyshev types inequalities involving integral operators. J. Math. Inequal. 10(2), 491-504 (2020)

32. Pólya, G., Szegö, G.: Aufgaben und Lehrsatze aus der analysis, Band 1, Die Grundlehren der mathematischen Wissenschaften 19. Springer, Berlin (1925)

33. Qi, F., Rahman, G., Hussain, S.M., Du, W.S., Nisar, K.S.: Some inequalities of Čebyšev type for conformable k-fractional integral operators. Symmetry 10, 614 (2018). https://doi.org/10.3390/sym10110614

34. Qi, H., Yussouf, M., Mehmood, S., Chu, Y.M., Farid, G.: Fractional integral versions of Hermite-Hadamard type inequality for generalized exponentially convexity. AIMS Math. 5(6), 6030-6042 (2020)

35. Rahman, G., Abdeljawad, T., Jarad, F., Khan, A., Nisar, K.S.: Certain inequalities via generalized proportional Hadamard fractional integral operators. Adv. Differ. Equ. 2019, 454 (2019). https://doi.org/10.1186/s13662-019-2381-0

36. Rahman, G., Abdeljawad, T., Khan, A., Nisar, K.S.: Some fractional proportional integral inequalities. J. Inequal. Appl. 2019, 244 (2019). https://doi.org/10.1186/s13660-019-2199-z

37. Rahman, G., Khan, A., Abdeljawad, T., Nisar, K.S.: The Minkowski inequalities via generalized proportional fractional integral operators. Adv. Differ. Equ. 2019, 287 (2019). https://doi.org/10.1186/s13662-019-2229-7

38. Rahman, G., Nisar, K.S., Abdeljawad, T.: Tempered fractional integral inequalities for convex functions. Mathematics 8 , 500 (2020). https://doi.org/10.3390/math8040500

39. Rahman, G., Nisar, K.S., Abdeljawad, T.: Certain Hadamard proportional fractional integral inequalities. Mathematics 8 , 504 (2020). https://doi.org/10.3390/math8040504

40. Rahman, G., Nisar, K.S., Abdeljawad, T., Ullah, S.: Certain fractional proportional integral inequalities via convex functions. Mathematics 8, 222 (2020). https://doi.org/10.3390/math8020222

41. Rahman, G., Nisar, K.S., Ghaffar, A., Qi, F.: Some inequalities of the Grüss type for conformable k-fractional integral operators. Rev. R. Acad. Cienc. Exactas Fís. Nat., Ser. A Mat. 114, 9 (2020). https://doi.org/10.1007/s13398-019-00731-3

42. Rahman, G., Nisar, K.S., Mubeen, S., Choi, J.: Certain Inequalities involving the $(k, \rho)$-fractional integral operator. Far East J. Math. Sci.: FJMS 103(11), 1879-1888 (2018)

43. Rahman, G., Nisar, K.S., Qi, F.: Some new inequalities of the Gruss type for conformable fractional integrals. AlMS Math. 3(4), 575-583 (2018)

44. Rahman, G., Nisar, K.S., Rashid, S., Abdeljawad, T.: Certain Grüss-type inequalities via tempered fractional integrals concerning another. J. Inequal. Appl. 2020, 147 (2020). https://doi.org/10.1186/s13660-020-02420-x

45. Rahman, G., Ullah, Z., Khan, A., Set, E., Nisar, K.S.: Certain Chebyshev type inequalities involving fractional conformable integral operators. Mathematics 7, 364 (2019). https://doi.org/10.3390/math7040364

46. Rahmnan, G., Abdeljawad, T., Jarad, F., Nisar, K.S.: Bounds of generalized proportional fractional integrals in general form via convex functions and their applications. Mathematics 8, 113 (2020). https://doi.org/10.3390/math8010113

47. Rashid, S., Jarad, F., Chu, Y.M.: A note on reverse Minkowski inequality via generalized proportional fractional integral operator with respect to another function. Math. Probl. Eng. 2020, Article ID 7630260 (2020)

48. Rashid, S., Jarad, F., Noor, M.A., Kalsoom, H., Chu, Y.M.: Inequalities by means of generalized proportional fractional integral operators with respect to another function. Mathematics 7(12), 1225 (2019). https://doi.org/10.3390/math7121225

49. Samko, S.G., Kilbas, A.A., Marichev, O.I.: Fractional Integrals and Derivatives, Theory and Applications. Gordon \& Breach, Yverdon (1993) Edited and with a foreword by S. M. Nikol'skì, Translated from the 1987 Russian original, Revised by the authors

50. Sarikaya, M.Z., Budak, H.: Generalized Ostrowski type inequalities for local fractional integrals. Proc. Am. Math. Soc. $145(4), 1527-1538(2017)$

51. Sarikaya, M.Z., Dahmani, Z., Kiris, M.E., Ahmad, F.: (k,s)-Riemann-Liouville fractional integral and applications. Hacet. J. Math. Stat. 45(1), 77-89 (2016)

52. Set, E., Tomar, M., Sarikaya, M.Z.: On generalized Grüss type inequalities for k-fractional integrals. Appl. Math. Comput. 269, 29-34 (2015)

53. Tassaddiq, A., Rahman, G., Nisar, K.S., Samraiz, M.: Certain fractional conformable inequalities for the weighted and the extended Chebyshev functionals. Adv. Differ. Equ. 2020, 96 (2020). https://doi.org/10.1186/s13662-020-2543-0

54. Yildirim, H., Kirtay, Z:: Ostrowski inequality for generalized fractional integral and related inequalities. Malaya J. Mat. 2, 322-329 (2014)

55. Zhou, S.S., Rashid, S., Jarad, F., Kalsoom, H., Chu, Y.M.: New estimates considering the generalized proportional Hadamard fractional integral operators. Adv. Differ. Equ. 2020, 275 (2020).

https://doi.org/10.1186/s13662-020-02730-w 